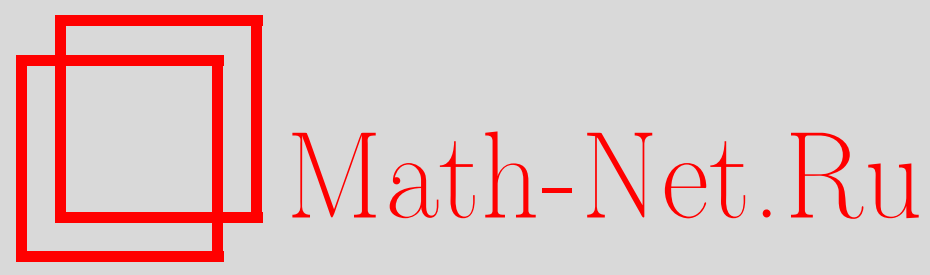

Ю. В. Нестеренко, Об одном тождестве Малера, Матем. заметки, 2006, том 79, выпуск 1, 107-119

DOI: https://doi.org/10.4213/mzm2679

Использование Общероссийского математического портала Math-Net.Ru подразумевает, что вы прочитали и согласны с пользовательским соглашением http://www . mathnet.ru/rus/agreement

Параметры загрузки:

IP : 54.92.164.108

26 апреля 2023 г., 18:05:27

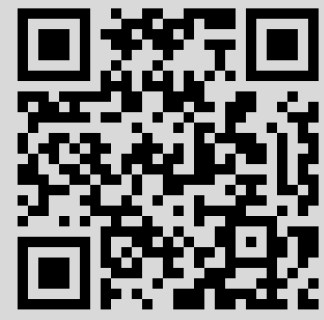




\section{ОБ ОДНОМ ТОЖДЕСТВЕ МАЛЕРА}

\section{Ю.В. Нестеренко}

Доказьваются представления некоторых кратных интегралов, зависящих от комплексного параметра $z$, в виде многочленов от $z$ и $\ln (1-z)$. Подобные тождества впервые использовал K. Малер в связи с доказательствами некоторых результатов теории трансцендентных чисел.

Библиография: 13 названий.

Введение. В настоящей статье рассматриваются некоторые свойства функций, задаваемых интегралами

$$
F(z)=\int_{[0,1]^{m}} \prod_{i=1}^{m} \frac{x_{i}^{a_{i}-1}\left(1-x_{i}\right)^{b_{i}-a_{i}-1}}{\left(1-z x_{i} \cdots x_{m}\right)^{c_{i}}} d x_{1} \cdots d x_{m}
$$

где $a_{i}, b_{i}, c_{i}-$ фиксированные действительные числа. Будет предполагаться, что $z-$ комплексное число, $|z|<1$, и $0<a_{i}<b_{i}$. Это обеспечивает сходимость. Интегралы такого вида используются в теории трансцендентных чисел для конструкции совместных диофантовых приближений к значениям гипергеометрических функций, в частности, логарифмической функции и бинома. С их помощью получаются также совместные приближения к значениям полилогарифмов и так называемьх обобщенных полилогарифмов.

Пусть $\bar{s}=\left(s_{1}, \ldots, s_{m}\right)$ - вектор с целыми положительньци координатами. Обобщенными полилогарифмами будем назьвать функции, задаваемые следующими кратньми рядами:

$$
\operatorname{Li}_{\bar{s}}(z)=\sum_{n_{1}>\cdots>n_{m} \geqslant 1} \frac{z^{n_{1}}}{n_{1}^{s_{1}} \cdots n_{m}^{s_{m}}}, \quad \operatorname{Le}_{\bar{s}}(z)=\sum_{n_{1} \geqslant \cdots \geqslant n_{m} \geqslant 1} \frac{z^{n_{1}}}{n_{1}^{s_{1}} \cdots n_{m}^{s_{m}}}
$$

При $m=1$ таким способом получаются классические полилогарифмы. Свойства обобщенных полилогарифмов изучались в статьях различных авторов (см. [1] для дальнейших ссылок). Функции каждого из семейств Li и Le линейно независимы над $\mathbb{C}(z)$, а линейные пространства над $\mathbb{Q}$, порождаемые этими семействами, совпадают. Явные

Работа вьполнена при частичной поддержке Фонда Александра фон Гумбольдта и Российского фонда фундаментальных исследований, грант № 03-01-00359. 
формулы, выражающие функции одного семейства через функции другого, указаны в [2, предложения 2 и 3].

При целых значениях параметров $a_{i}, b_{i}, c_{i}$ интегралы вида $(1)$, умноженные на некоторую степень переменной $z$, представляются в виде линейной комбинации обобщенных полилогарифмов с полиномиальными от $z$ коэффициентами. Приведем некоторые примеры.

ПримеР 1. В работе В.Н. Сорокина [3] для доказательства иррациональности числа $\zeta(3)$ при любом целом неотрицательном $n$ устанавливается тождество

$$
\begin{aligned}
z^{2 n+1} \int_{[0,1]^{3}} \frac{\prod_{i=1}^{3} x_{i}^{n}\left(1-x_{i}\right)^{n}}{\left(1-z x_{2} x_{3}\right)^{n+1}\left(1-z x_{1} x_{2} x_{3}\right)^{n+1}} d x_{1} d x_{2} d x_{3} \\
=A_{0}(z)+A_{1}(z) \operatorname{Le}_{1}(z)+A_{2}(z) \operatorname{Le}_{1,1}(z)+A_{3}(z) \operatorname{Le}_{2,1}(z),
\end{aligned}
$$

где $A_{i}(z)$ - многочлены с рациональными коэффициентами степени не вьше $n$. При этом интеграл сходится в точке $z=1$, и вьполняются равенства $A_{1}(1)=A_{2}(1)=0$. Поскольку $\mathrm{Le}_{2,1}(1)=2 \zeta(3)$, таким способом получаются рациональные приближения Апери к $\zeta(3)$.

ПримеР 2. Для оценки меры трансцендентности числа $\pi^{2}$ Сорокин в работе [4] доказывает, что

$$
\begin{aligned}
& z^{(r+1)(n+1)-1} \int_{[0,1]^{2 r}} \prod_{i=1}^{r} \frac{x_{2 i-1}^{i(n+1)-1}\left(1-x_{2 i-1}\right)^{n} x_{2 i}^{i(n+1)-1}\left(1-x_{2 i}\right)^{n}}{\left(1-z x_{2 i-1} x_{2 i} \cdots x_{2 r-1} x_{2 r}\right)^{n+1}} d x_{1} \cdots d x_{2 r} \\
& =A_{0}(z)+\sum_{i=1}^{r}\left(A_{i}(z) \operatorname{Li}_{\{2\}_{i}}(z)+B_{i}(z) \operatorname{Li}_{1\{2\}_{i-1}}(z)\right)
\end{aligned}
$$

где $\{2\}_{i}=(2, \ldots, 2) \in \mathbb{Z}^{i}, A_{i}(z), B_{i}(z)$ - многочлены с рациональными коэффициентами степени не вьше $n$. При этом также выполняются равенства $B_{i}(1)=0$.

ПримеР 3. В случае

$$
c_{2}=\cdots=c_{m}=0, \quad 1 \leqslant a_{1} \leqslant \ldots a_{m}<b_{m} \leqslant \cdots \leqslant b_{1}, a_{1} \leqslant c_{1} \leqslant \sum_{i=1}^{m}\left(b_{i}-a_{i}\right)
$$

имеет место представление

$$
\begin{aligned}
z^{b_{1}-1} & \int_{[0,1]^{m}} \frac{\prod_{i=1}^{m} x_{i}^{a_{i}-1}\left(1-x_{i}\right)^{b_{i}-a_{i}-1}}{\left(1-z x_{1} \cdots x_{m}\right)^{c_{1}}} d x_{1} \cdots d x_{m} \\
= & A_{0}(z)+A_{1}(z) \operatorname{Li}_{1}(z)+\cdots+A_{m}(z) \operatorname{Li}_{m}(z),
\end{aligned}
$$

где $A_{k}(z)$ - многочлены от $z$ с рациональньпи коэффициентами, причем $\operatorname{deg} A_{i}(z) \leqslant$ $b_{1}-a_{i}-1,1 \leqslant i \leqslant m, \operatorname{deg} A_{0}(z) \leqslant b_{1}-a_{1}-1$ (см. [5]). При специальном выбоpe $c_{1}=(2 r+1) n+2, a_{i}=r n+1, b_{i}=(r+1) n+2$, где $r$ - некоторое целое число, $1 \leqslant 2 r+1 \leqslant m$, это тождество использовалось Т. Ривоалем в работе [6] для доказательства бесконечности множества иррациональных чисел в последовательности $\zeta(2 k+1)$, $k=1,2, \ldots$. 
ПримеР 4. Следующее тождество найдено С.А. Злобиным [7]. Если параметры $a_{i}, b_{i}, c_{i}$ - целые положительные числа с условиями

$$
a_{i}<b_{i}, \quad \sum_{j=m-i+1}^{m}\left(b_{j}-a_{j}-c_{j}\right) \geqslant 0, \quad b_{i} \leqslant a_{i-1}+c_{i}
$$

при всех возможных значениях индекса $i$, то

$$
z^{b-1} F(z)=A_{0}(z)+\sum_{k=1}^{m} A_{k}(z) \operatorname{Le}_{\{1\}_{k}}(z)
$$

где $b=\max b_{j}$ и $A_{k}(z)$ - многочлены с рациональными коэффициентами, степени которых оцениваются некоторыми величинами, зависяшими от параметров $a_{i}, b_{i}, c_{i}$.

ПРимеР 5. Злобину принадлежит общий результат (см. [8], [7]). Пусть $a_{i}, b_{i}, c_{i}-$ неотрицательные целые числа с условиями

$$
b_{i}>a_{i}>0, \quad \sum_{i=k}^{m}\left(b_{i}-a_{i}-c_{i}\right) \geqslant 0, \quad 1 \leqslant k \leqslant m .
$$

Обозначим $a=\min a_{i}, b=\max b_{i}$. В этих условиях функция $z^{b-1} F(z)$ может быть представлена в виде линейной формы от 1 и обобщенных полилогарифмов $\operatorname{Le}_{\bar{s}}(z), \bar{s}=$ $\left(s_{1}, \ldots, s_{l}\right)$, где $l$ не превосходит количества положительных чисел среди $c_{i}$, и вьполняется неравенство $s_{1}+\cdots+s_{l} \leqslant m$. Коэффициенты этой линейной формы есть многочлены с рациональными коэффициентами, степень которых не превосходит $b-a-1$.

Примеры 1-4 показьвают, что последнее утверждение не всегда точно отражает реальное положение вещей. Вместе с тем, знание того, какие полилогарифмы действительно входят в линейную форму, а также того, каковы действительные величины степеней многочленов $A_{j}(z)$, весьма важно для теоретико-числовых приложений. Конечно, важны и арифметические характеристики многочленов - величина их коэффициентов и правильные оценки их общих знаменателей, но в настоящей статье мы не будем затрагивать арифметические вопросы.

Ниже будут установлены достаточно точные ограничения на параметры $a_{i}, b_{i}, c_{i}$, при которых интеграл (1) представим в виде линейной комбинации функций $\mathrm{Li}_{\{1\}_{k}}$.

1. Интегральное тождество Малера. Вероятно, первым частные интегралы вида (1) в связи с диофантовыми приближениями корней из рациональных чисел стал рассматривать в 1931 г. К. Малер (см. [9]). Впрочем, его интегралы отличались от (1) и могут быть получены из них заменой переменных $x_{j}=t_{j} / t_{j+1}, j=1, \ldots, m-1$, $x_{m}=t_{m}$; соответственно область интегрирования у Малера задавалась неравенствами $0 \leqslant t_{1} \leqslant \cdots \leqslant t_{m} \leqslant 1$. В случае Малера интегралы (1) представлялись в виде линейных форм от биномиальных функций $(1-z)^{\omega_{i}}$ с показателями $\omega_{i}$, не сравнимьми по модулю 1. Малер нашел также представление этих линейньх форм в виде комплексных контурных интегралов. Формулы Малера воспроизведены в работе А. Бейкера [10], где они использовались для доказательства оценок снизу линейных форм с целыми коэффициентами от корней из алгебраических чисел. Аналогичные формулы для экспоненциальной функции вместо бинома можно найти в работе Малера [11].

В этом пункте будет доказана следующая теорема. 
Теорема 1. Пусть $\omega_{0}, \ldots, \omega_{m} \in \mathbb{C} u \rho_{0}, \ldots, \rho_{m}$ - иелые положительные числа. Тогда для любого $z \in \mathbb{C},|\arg (1-z)|<\pi$, справедливо тождество

$$
\begin{gathered}
\frac{(-z)^{\rho_{0}+\cdots+\rho_{m}-1}}{\prod_{j=0}^{m} \Gamma\left(\rho_{j}\right)} \int_{[0,1]^{m}} \prod_{j=1}^{m} \frac{x_{j}^{\rho_{0}+\cdots+\rho_{j-1}-1}\left(1-x_{j}\right)^{\rho_{j}-1}}{\left(1-z x_{j} \cdots x_{m}\right)^{\rho_{j}+\omega_{j}-\omega_{j-1}}} d x_{1} \cdots d x_{m} \\
=\frac{1}{2 \pi i} \int_{C} \prod_{j=0}^{m} \frac{\Gamma\left(\zeta-\omega_{j}-\rho_{j}+1\right)}{\Gamma\left(\zeta-\omega_{j}+1\right)}(1-z)^{\zeta-\omega_{m}} d \zeta
\end{gathered}
$$

әде $(1-z)^{\zeta}=e^{\zeta \log (1-z)} u \log (1-z)=\log |1-z|+i \arg (1-z)$, другие комплексные степени 1-z определяются точно так же, а $C$ - гладкий замкнутый контур, однократно обходящий в положительном направлении все точки $\omega_{j}+\sigma, 0 \leqslant \sigma<\rho_{j}$, $\sigma \in \mathbb{Z}$. В случае $m=0$ интеграл в левой части считается равным 1.

Это утверждение в случае $\omega_{h}-\omega_{k} \notin \mathbb{Z}$ и с точностью до замены переменных в кратном интеграле по существу было доказано в 1931 г. Малером [9]. Ясно, что общий случай может быть получен с помощью аналитического продолжения по переменньп $\omega_{h}$. Малер доказал, что оба интеграла в левой и правой части (2) равны линейным формам от биномов $(1-z)^{\omega_{h}}$, коэффициенты при которых суть многочлены от $z$ степени $\rho_{h}-1$, и эти линейные формы являются приближениями Паде для указанной совокупности биномов. Единственность приближения Паде означала совпадение этих линейных форм, а следовательно, и интегралов.

Оба интегральных представления использовались Малером для выяснения различных аналитических и арифметических свойств построенных линейных форм. Но в случае $\omega_{j} \in \mathbb{Z}$ применялся только комплексньй интеграл.

Обозначим $v_{j}=\rho_{j}+\omega_{j}, j=0,1, \ldots, m$. Можно считать, что интегралы, присутствующие в теореме 1 , являются функциями параметров $\omega_{j}, v_{j}$.

СлЕДСТВИЕ 1. Кратный интеграл (2) не меняется при сдвиге всех параметров $\omega_{j}$ на одно и то же произвольное число. Будучи умножсенным на $(1-z)^{\omega_{m}}$, он не меняется, как при любой перестановке параметров $\omega_{j}$, так и при любой перестановке параметров $v_{j}$, при которой вновь полученные интегралы сходятся.

ДокАЗАТЕльство. Первое утверждение следует из определения кратного интеграла (2). Будучи умноженным на $(1-z)^{\omega_{m}}$ комплексньй интеграл в $(2)$, очевидно, симметричен относительно двух групп переменных $v_{j}$ и $\omega_{j}$. Это доказьвает второе утверждение.

Заметим, что комплексньй интеграл в правой части $(2)$, умноженньй на $(1-z)^{\omega_{m}}$, может быть представлен как $G$-функция Мейера

$$
G_{m+1, m+1}^{0, m+1}\left(\begin{array}{c|c}
\omega_{0}+\rho_{0}, \ldots, \omega_{m}+\rho_{m} & 1-z \\
\omega_{0}, \ldots, & \omega_{m}
\end{array}\right)
$$

(см. [12]). Такая форма записи в связи с построением приближений Эрмита-Паде для биномов и степеней логарифмов использовалась Х. Ягером (см. [13, части IV и VI]).

Предположим, что все параметры $\omega_{j}$ суть целые числа. В дальнейшем удобно использовать обозначения $\mathcal{T}_{j}=\left[\omega_{j}, \omega_{j}+\rho_{j}-1\right], 0 \leqslant j \leqslant m$, для отрезков действительной прямой и $\mathcal{T}=\bigcup_{j=0}^{m} \mathcal{T}_{j}$. Пусть также для каждого $l \in \mathbb{Z}$ символ $d(l)$ обозначает количество отрезков $\mathcal{T}_{j}$, содержаших точку $l$. 
СлЕДСТВИЕ 2. Если все параметры $\omega_{0}, \ldots, \omega_{m}$ суть иелые числа, причем $\omega_{j} \geqslant$ $\omega_{m}=0, j=0,1, \ldots, m-1$, то кратный интеграл в (2) равен

$$
\sum_{k=0}^{d-1} A_{k}(z) \log ^{k}(1-z), \quad A_{k}(z) \in \mathbb{Q}[z]
$$

где $d=\max _{l \in \mathcal{T}} d(l)$. При этом степень многочлена $A_{k}(z)$ не превосходит максимального из чисел $l$ множсества $\mathcal{T}$ с условием $d(l) \geqslant k+1$, а кратность, с которой этот многочлен обращается в нуль в точке $z=1$, не меньше минимального из чисел $l$ с тем же условием.

ДокАЗАТЕЛЬСТво. Для рациональной функции

$$
R(\zeta)=\prod_{j=0}^{m} \frac{\Gamma\left(\zeta-\omega_{j}-\rho_{j}+1\right)}{\Gamma\left(\zeta-\omega_{j}+1\right)}=\frac{1}{\prod_{j=0}^{m}\left(\zeta-\omega_{j}-\rho_{j}+1\right) \cdots\left(\zeta-\omega_{j}\right)}
$$

справедливо представление

$$
R(\zeta)=\prod_{j \in \mathcal{T}} \frac{1}{(\zeta-j)^{d(j)}}
$$

Согласно теореме о вычетах комплексньй интеграл в правой части равенства (2) равен

$$
\begin{aligned}
& \sum_{l \in \mathcal{T}} \operatorname{Res}_{\zeta=l}\left(R(\zeta)(1-z)^{\zeta}\right)=\sum_{l \in \mathcal{T}}\left(\prod_{\substack{j \in \mathcal{T} \\
j \neq l}}(\zeta-j)^{-d(j)}(1-z)^{\zeta}\right)_{\zeta=l}^{(d(l)-1)} \\
& \quad=\sum_{l \in \mathcal{T}} \sum_{k=0}^{d(l)-1}\left(\begin{array}{c}
d(l)-1 \\
k
\end{array}\right)(1-z)^{l} \log ^{k}(1-z)\left(\prod_{\substack{j \in \mathcal{T} \\
j \neq l}}(\zeta-j)^{-d(j)}\right)_{\zeta=l}^{(d(l)-1-k)} \\
& \quad=\sum_{k=0}^{d-1} A_{k}(z) \log ^{k}(1-z)
\end{aligned}
$$

где $d=\max _{l \in \mathcal{T}} d(l)$ и

$$
A_{k}(z)=\sum_{\substack{l \in \mathcal{T} \\ d(l) \geqslant k+1}} \gamma_{l, k}(1-z)^{l}, \quad \gamma_{l, k} \in \mathbb{Q} .
$$

Отсюда следует требуемое утверждение.

Для доказательства теоремы 1 понадобятся две простые леммы.

Лемма 1. Пусть $n$ - иелое неотрицательное число, $f(z)$ - функиия, непрерывная в окрестности точки $z=0, u$

$$
g(z)=z^{n+1} \int_{0}^{1} \frac{(1-x)^{n}}{n !} f(z x) d x
$$

Tогда $g^{(n+1)}(z)=f(z)$. 
ДокАЗАТЕЛЬСТВО. При $n \geqslant 0$ справедливо представление

$$
g(z)=\int_{0}^{z} \frac{(z-t)^{n}}{n !} f(t) d t
$$

из которого следует нужное утверждение при $n=0$. Дифференцируя (3) при $n \geqslant 1$, получаем равенство

$$
g^{\prime}(z)=\int_{0}^{z} \frac{(z-t)^{n-1}}{(n-1) !} f(t) d t
$$

позволяющее индукцией по $n$ вьвести необходимое утверждение и в общем случае.

Пусть $a_{i}, b_{i} \in \mathbb{Z}, c_{i} \in \mathbb{C}, i=1, \ldots, m$, причем $b_{i}>a_{i}>0$. Определим для каждого целого $r, 1 \leqslant r \leqslant m$, функцию

$$
F_{r}(z)=\frac{z^{b_{r}-1}}{\prod_{j=1}^{r} \Gamma\left(b_{j}-a_{j}\right)} \int_{[0,1]^{r}} \prod_{j=1}^{r} \frac{x_{j}^{a_{j}-1}\left(1-x_{j}\right)^{b_{j}-a_{j}-1}}{\left(1-z x_{j} \cdots x_{r}\right)^{c_{j}}} d x_{1} \cdots d x_{r}
$$

и положим также $F_{0}(z)=1$. Здесь считается, что $z \in \mathbb{C},|\arg (1-z)|<\pi$, и комплексные степени определяются с условием указанного ранее соглашения. Для краткости буквой $D$ будем обозначать символ производной $d / d z$.

ЛЕмма 2. Во введенных выше обозначениях выполняется равенство

$$
D^{b_{r}-a_{r}} F_{r}(z)=\frac{z^{a_{r}-b_{r-1}}}{(1-z)^{c_{r}}} \cdot F_{r-1}(z), \quad r=1, \ldots, m,
$$

əде считается $b_{0}=1$.

ДокАЗАТЕЛЬСТво. Из определения функций $F_{j}(z)$ находим тождество

$$
F_{r}(z)=z^{b_{r}-a_{r}} \int_{0}^{1} \frac{\left(1-x_{r}\right)^{b_{r}-a_{r}-1}}{\left(b_{r}-a_{r}-1\right) !} \frac{\left(z x_{r}\right)^{a_{r}-b_{r-1}}}{\left(1-z x_{r}\right)^{c_{r}}} F_{r-1}\left(z x_{r}\right) d x_{r}
$$

из которого в силу леммы 1 следует утверждаемое равенство.

ДоКАЗАТЕЛЬСТВо ТЕОРЕМЫ 1. Докажем тождество (2) индукцией по $m$. При $m=0$ имеем

$$
\begin{aligned}
& \frac{1}{2 \pi i} \int_{C} \frac{\Gamma\left(\zeta-\omega_{0}-\rho_{0}+1\right)}{\Gamma\left(\zeta-\omega_{0}+1\right)}(1-z)^{\zeta-\omega_{0}} d \zeta=\frac{1}{2 \pi i} \int_{C} \frac{(1-z)^{\zeta-\omega_{0}}}{\prod_{\sigma=0}^{\rho_{0}-1}\left(\zeta-\omega_{0}-\sigma\right)} d \zeta \\
& \quad=\sum_{\sigma=0}^{\rho_{0}-1} \frac{(1-z)^{\sigma}(-1)^{\rho_{0}-1-\sigma}}{\sigma !\left(\rho_{0}-1-\sigma\right) !}=\frac{(-1)^{\rho_{0}-1}}{\left(\rho_{0}-1\right) !} \sum_{\sigma=0}^{\rho_{0}-1}\left(\begin{array}{c}
\rho_{0}-1 \\
\sigma
\end{array}\right)(z-1)^{\sigma}=\frac{(-z)^{\rho_{0}-1}}{\left(\rho_{0}-1\right) !}
\end{aligned}
$$

Получившееся выражение совпадает с левой частью (2) при $m=0$.

Пусть теперь $m \geqslant 1$ и для кратных интегралов размерности меньшей $m$ утверждение теоремы доказано. Определим функции $F_{r}(z)$, как это указано ранее, выбрав параметры следующим образом:

$$
a_{j}=\rho_{0}+\cdots+\rho_{j-1}, \quad b_{j}=\rho_{0}+\cdots+\rho_{j}, \quad c_{j}=\rho_{j}+\omega_{j}-\omega_{j-1}, \quad 1 \leqslant j \leqslant m .
$$


Тогда, очевидно, левая часть равенства $(2)$ имеет вид $(-1)^{\rho_{0}+\cdots+\rho_{m}-1} F_{m}(z)$.

Определим теперь функции

$$
I_{r}(z)=\frac{(-1)^{\rho_{0}+\cdots+\rho_{r}-1}}{2 \pi i} \int_{C} \prod_{j=0}^{r} \frac{\Gamma\left(\zeta-\omega_{j}-\rho_{j}+1\right)}{\Gamma\left(\zeta-\omega_{j}+1\right)}(1-z)^{\zeta-\omega_{m}} d \zeta
$$

для $0 \leqslant r<m$. Тогда равенство $(2)$ принимает вид $F_{m}(z)=I_{m}(z)$.

Дифференцируя функцию $I_{m}(z)$ под знаком интеграла, находим

$$
\begin{aligned}
D^{\rho_{m}} I_{m}(z) & =\frac{(-1)^{\rho_{0}+\cdots+\rho_{m-1}-1}}{2 \pi i} \int_{C} \prod_{j=0}^{m-1} \frac{\Gamma\left(\zeta-\omega_{j}-\rho_{j}+1\right)}{\Gamma\left(\zeta-\omega_{j}+1\right)}(1-z)^{\zeta-\omega_{m}-\rho_{m}} d \zeta \\
& =\frac{1}{(1-z)^{\rho_{m}+\omega_{m}-\omega_{m-1}}} I_{m-1}(z) .
\end{aligned}
$$

В точности такое же соотношение

$$
D^{\rho_{m}} F_{m}(z)=\frac{1}{(1-z)^{\rho_{m}+\omega_{m}-\omega_{m-1}}} F_{m-1}(z)
$$

получается, если к функциям $F_{m}(z)$ и $F_{m-1}(z)$ применить лемму 2 . По предположению индукции вьполняется равенство $F_{m-1}(z)=I_{m-1}(z)$, откуда следует $D^{\rho_{m}} F_{m}(z)=$ $D^{\rho_{m}} I_{m}(z)$, т.е. разность $F_{m}(z)-I_{m}(z)$ есть многочлен степени меньшей $\rho_{m}$.

Согласно определению $F_{m}(z)$ справедливо неравенство

$$
\operatorname{ord}_{z=0} F_{m}(z) \geqslant \rho_{0}+\cdots+\rho_{m}-1 \text {. }
$$

Докажем, что и для функции $I_{m}(z)$ выполняется такая же оценка кратности нуля в начале координат. Если $z$ достаточно мало (малость зависит от контура $C$ ), то

$$
(1-z)^{\zeta-\omega_{m}}=e^{\left(\zeta-\omega_{m}\right) \log (1-z)}=\sum_{n=0}^{\infty} \frac{\left(\zeta-\omega_{m}\right)^{n}}{n !}(\log (1-z))^{n}
$$

и последний ряд равномерно сходится на $C$. Поэтому

$$
I_{m}(z)=\sum_{n=0}^{\infty} \frac{(\log (1-z))^{n}}{n !} \cdot \frac{1}{2 \pi i} \int_{C}\left(\zeta-\omega_{m}\right)^{n} \cdot \prod_{j=0}^{m} \frac{\Gamma\left(\zeta-\omega_{j}-\rho_{j}+1\right)}{\Gamma\left(\zeta-\omega_{j}+1\right)} d \zeta
$$

Учитьвая, что

$$
\left(\zeta-\omega_{m}\right)^{n} \cdot \prod_{j=0}^{m} \frac{\Gamma\left(\zeta-\omega_{j}-\rho_{j}+1\right)}{\Gamma\left(\zeta-\omega_{j}+1\right)}=\frac{\left(\zeta-\omega_{m}\right)^{n}}{\prod_{j=0}^{m}\left(\zeta-\omega_{j}\right) \cdots\left(\zeta-\omega_{j}-\rho_{j}+1\right)}
$$

есть рациональная функция, имеющая в $\infty$ нуль порядка $\rho_{0}+\cdots+\rho_{m}-n$, заключаем, что в сумме $(5)$ все слагаемые с $n \leqslant \rho_{0}+\cdots+\rho_{m}-2$ равны нулю. Это в силу равенства $\operatorname{ord}_{z=0} \log (1-z)=1$ завершает доказательство оценки для кратности нуля $I_{m}(z)$ и доказьвает тем самым равенство $F_{m}(z)=I_{m}(z)$. Теорема 1 доказана. 
2. Линейные формы от степеней логарифмов. Кратные интегралы в тождестве Малера имеют вид (1) при $b_{j}=a_{j+1}$. Ниже будет доказан аналог следствия 2 при менее ограничительных предположениях о параметрах $a_{i}, b_{i}, c_{i}$.

Рассмотрим последовательность функций

$$
f_{0}(z)=1, \quad f_{k}(z)=\frac{1}{k !}(-\log (1-z))^{k}, \quad k=1,2, \ldots
$$

Эти функции связаны между собой соотношениями

$$
f_{k}^{\prime}(z)=\frac{1}{1-z} f_{k-1}(z)
$$

Легко проверить, что $\operatorname{Li}_{\{1\}_{k}}(z)=f_{k}(z)$.

Теорема 2. Пусть $a_{1}, b_{1}, c_{1}, \ldots, a_{m}, b_{m}, c_{m}$ - такие целые числа, что

$$
1 \leqslant a_{1}<b_{1} \leqslant a_{2}<b_{2} \leqslant \cdots \leqslant a_{m}<b_{m}, \quad c_{i} \leqslant b_{i}-a_{i}, \quad i=1, \ldots, m .
$$

Тогда в области $z \in \mathbb{C},|z|<1$, справедливо тождество

$$
\frac{z^{b_{m}-1}}{\prod_{i=1}^{m} \Gamma\left(b_{i}-a_{i}\right)} \int_{[0,1]^{m}} \prod_{i=1}^{m} \frac{x_{i}^{a_{i}-1}(1-x)^{b_{i}-a_{i}-1}}{\left(1-z x_{i} \cdots x_{m}\right)^{c_{i}}} d x_{1} \cdots d x_{m}=\sum_{j=0}^{d} A_{j}(z) f_{j}(z),
$$

где $d$ не превосходит количества положительных чисел в наборе $c_{i}, 1 \leqslant i \leqslant m, a$ многочлены $A_{j}(z) \in \mathbb{Q}[z], 0 \leqslant j \leqslant d$, удовлетворяют условиям

$\operatorname{deg} A_{j}(z) \leqslant b_{m}-\min _{0 \leqslant k \leqslant m}\left(a_{k}+\sum_{i=k+1}^{m} c_{i}\right)-1, \quad \operatorname{ord}_{z=1} A_{j}(z) \geqslant \sum_{i=m+1-j}^{m}\left(b_{i}-a_{i}-c_{i}\right)$.

Здесь предполагается, что $a_{0}$ и пустая сумма в последнем неравенстве равны 0. Неравенства $0<a_{j}<b_{j}$ нужны для обеспечения сходимости кратного интеграла.

В основе доказательства теоремы 2 лежит следующее утверждение.

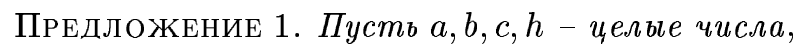

$$
0<a<b, \quad c \leqslant b-a, \quad h \geqslant 0 .
$$

Тогда в области $z \in \mathbb{C},|z|<1$, выполняется тождество

$$
\frac{z^{b-1}}{(b-a-1) !} \int_{0}^{1} \frac{x^{a-1}(1-x)^{b-a-1}}{(1-z x)^{c}} f_{h}(z x) d x=\sum_{k=0}^{h+1} A_{k}(z) f_{k}(z)
$$

әде $A_{k}(z) \in \mathbb{Q}[z]$, причем

$$
\begin{aligned}
\operatorname{deg} A_{h+1}(z) \leqslant & b-a-1, \quad \operatorname{deg} A_{k}(z) \leqslant b-\min (a, c)-1, \quad 0 \leqslant k \leqslant h \\
& \operatorname{ord}_{z=1} A_{k}(z) \geqslant b-a-c, \quad 1 \leqslant k \leqslant h+1 .
\end{aligned}
$$

При $c \leqslant 0$ многочлен $A_{h+1}(z)$ равен 0.

Для доказательства этого утверждения понадобятся две леммы. 
ЛЕмма 3. При любом иелом $r \geqslant 0$ справедливо равенство

$$
f_{k}^{(r)}(z)=\frac{1}{(1-z)^{r}} \sum_{j=0}^{k} c(k, r, j) f_{j}(z)
$$

әде $c(k, r, j)$ - некоторые челые числа. При $r \geqslant 1$ суммирование можно вести только до $k-1$.

ДокАЗАТЕЛЬСТво. Лемма легко доказьвается индукцией по $r$. При $r=0$ равенство, очевидно, вьполняется. Шаг индукции проверяется с помощью (6) и простых вычислений:

$$
\begin{aligned}
f_{k}^{(r+1)}(z) & =\frac{d}{d z}\left(\frac{1}{(1-z)^{r}} \sum_{j=0}^{k} c(k, r, j) f_{j}(z)\right) \\
& =\frac{1}{(1-z)^{r+1}} \sum_{j=0}^{k}(r c(k, r, j)+c(k, r, j+1)) f_{j}(z) .
\end{aligned}
$$

Здесь $c(k, r, k+1)=0$. Соотношение (9) доказьвает лемму.

ДоКАЗАТЕЛЬСТВО ПРЕДЛОЖЕНИЯ 1 . В дальнейшем будем считать, что целые числа $a, b, c, h$ удовлетворяют всем условиям предложения.

Определим два линейных пространства $\mathfrak{L}_{1}$ и $\mathfrak{L}_{2}$ над полем рациональных чисел. Пространство $\mathfrak{L}_{1}$ состоит из функций

$$
\sum_{k=0}^{h} B_{k}(z) f_{k}(z), \quad B_{k}(z) \in \mathbb{Q}[z], \quad \operatorname{deg} B_{k}(z) \leqslant \max (a, c)-1,
$$

а пространство $\mathfrak{L}_{2}$ из функций

$$
\begin{gathered}
\sum_{k=0}^{h+1} A_{k}(z) f_{k}(z), \quad A_{k}(z) \in \mathbb{Q}[z], \\
\operatorname{deg} A_{h+1}(z) \leqslant b-a-1, \quad \operatorname{deg} A_{k}(z) \leqslant b-\min (a, c)-1, \quad k=0, \ldots, h, \\
\operatorname{ord}_{z=1} A_{j}(z) \geqslant b-a-c, \quad j=1, \ldots, h+1, \\
\operatorname{ord}_{z=1} A_{0}(z) \geqslant b-a-\min (0, c) .
\end{gathered}
$$

Заметим, что при $c \leqslant 0$ многочлен $A_{h+1}(z)$ у каждого элемента из $\mathfrak{L}_{2}$ равен 0.

Функция $\log (1-z)$ трансцендентна, поэтому $\operatorname{dim} \mathfrak{L}_{1}=(h+1) \max (a, c)$. Для того, чтобы вычислить размерность линейного пространства $\mathfrak{L}_{2}$, заметим, что совокупность многочленов $P \in \mathbb{Q}[z]$ с условиями $\operatorname{deg} P \leqslant u-1, \operatorname{ord}_{z=1} P \geqslant v$, где $u, v$ - целые числа, есть линейное пространство размерности $\max (0, u-v)$. Поэтому

$$
\begin{aligned}
\operatorname{dim} \mathfrak{L}_{2} & =h \max (a, c)+\max (0, a-\min (a, c)+\min (0, c))+\max (0, c) \\
& =h \max (a, c)+\max (\max (0, c), \max (a, c))=(h+1) \max (a, c)=\operatorname{dim} \mathfrak{L}_{1} .
\end{aligned}
$$

Рассмотрим отображение

$$
\varphi: f(z) \mapsto(1-z)^{c} f^{(b-a)}(z),
$$

определенное на $b-a$ раз дифференцируемых функциях. Так как ядро отображения $\varphi$ состоит из многочленов степени не вьше $b-a-1$, а все многочлены из $\mathfrak{L}_{2} \cap \mathbb{Q}[z]$ имеют в точке $z=1$ нуль кратности не меньше $b-a-\min (0, c), \operatorname{To} \operatorname{Ker}(\varphi) \cap \mathfrak{L}_{2}=(0)$. 
Лемма 4. Справедливо равенство

$$
\varphi\left(\mathfrak{L}_{2}\right)=\mathfrak{L}_{1}
$$

Поясним сначала, как из этой леммы следует предложение 1. Обозначим

$$
G(z)=\frac{z^{b-1}}{(b-a-1) !} \int_{0}^{1} \frac{x^{a-1}(1-x)^{b-a-1}}{(1-z x)^{c}} f_{h}(z x) d x .
$$

Согласно лемме 1 вьполняется равенство

$$
G^{(b-a)}(z)=\frac{z^{a-1}}{(1-z)^{c}} f_{h}(z)
$$

Оно может быть также переписано в виде

$$
\varphi(G(z))=z^{a-1} f_{h}(z) \in \mathfrak{L}_{1}
$$

По лемме 4 найдется функция $f(z) \in \mathfrak{L}_{2}$, для которой

$$
\varphi(f(z))=z^{a-1} f_{h}(z)
$$

и, значит, $G(z)-f(z) \in \operatorname{Ker}(\varphi)$. Учитывая, что $\operatorname{Ker}(\varphi)$ состоит из многочленов степени не выше $b-a-1 \leqslant b-\min (a, c)-1$, находим, что функция $G(z)$ представима в виде, утверждаемом предложением 1 .

ДоКАЗАТЕЛЬСтво ЛЕммы 4. Достаточно доказать включение $\varphi\left(\mathfrak{L}_{2}\right) \subset \mathfrak{L}_{1} ;$ нужное равенство следует из этого включения, равенства размерностей пространств $\mathfrak{L}_{1}, \mathfrak{L}_{2}$ (см. (13)) и $\operatorname{Ker}(\varphi) \cap \mathfrak{L}_{2}=(0)$.

В силу линейности отображения $\varphi$ для доказательства включения достаточно проверить, что

$$
\begin{aligned}
\varphi\left((1-z)^{k}\right) & \in \mathfrak{L}_{1}, & & b-a-\min (0, c) \leqslant k \leqslant b-\min (a, c)-1, \\
\varphi\left((1-z)^{k} f_{i}(z)\right) & \in \mathfrak{L}_{1}, & & b-a-c \leqslant k \leqslant b-\min (a, c)-1, \quad 1 \leqslant i \leqslant h, \\
\varphi\left((1-z)^{k} f_{h+1}(z)\right) & \in \mathfrak{L}_{1}, & & b-a-c \leqslant k \leqslant b-a-1 .
\end{aligned}
$$

Включение (14) выполняется, поскольку

$$
\varphi\left((1-z)^{k}\right)=(-1)^{b-a} \frac{k !}{(k-b+a) !}(1-z)^{k-(b-a-c)}
$$

есть многочлен степени не вьше $b-\min (a, c)-1-(b-a-c)=\max (a, c)-1$.

Включения (15) и (16) также проверяются непосредственным вычислением. Применяя правило Лейбница, находим

$$
\varphi\left((1-z)^{k} f_{i}(z)\right)=\sum_{r=0}^{b-a}(-1)^{r}\left(\begin{array}{c}
b-a \\
r
\end{array}\right) \frac{k !}{(k-r) !}(1-z)^{c+k-r} f_{i}^{(b-a-r)}(z)
$$


причем в последней сумме также должно вьполняться неравенство $r \leqslant k$. Применяя $\mathrm{K}$ производньп функции $f_{i}(z)$ представление из леммы 3 и пользуясь в случае (15) неравенствами $b-a-c \leqslant k \leqslant b-\min (a, c)-1$, легко получаем, что правая часть (17) есть линейная комбинация функций $f_{0}(z), \ldots, f_{i}(z), i \leqslant h$, с коэффициентами - многочленами от $z$ степени не вьше $\max (a, c)-1$. Это доказьвает включение $(15)$.

В случае (16) должно вьполняться неравенство $c \geqslant 1$. Кроме того, имеем $r \leqslant k \leqslant$ $b-a-1$. Поэтому порядок производных функции $f_{h+1}(z)$ в сумме $(17)$ будет не меньше 1. Значит, согласно лемме 3 правая часть (17) будет линейной комбинацией функций $f_{0}(z), \ldots, f_{h}(z)$. Неравенства $b-a-c \leqslant k \leqslant b-a-1$, как и в предыдушем случае, показывают, что коэффициенты этой линейной комбинации будут многочленами степени не вьше $c-1 \leqslant \max (a, c)-1$. Это доказьвает включение $(16)$.

Таким образом, лемма 4 , а вместе с ней и предложение 1 , доказаны.

ДоКАЗАТЕЛЬСТВо ТЕОРЕМЫ 2. НаПомним, что левая часть тождества из теоремы 2 обозначалась ранее как $F_{m}(z)$. Для доказательства мы воспользуемся индукцией по $m$.

При $m=1$ согласно предложению 1 (при $a=a_{1}, b=b_{1}, c=c_{1}, h=0$ ) вьполняется тождество

$$
F_{1}(z)=A_{0}(z)+A_{1}(z) f_{1}(z)
$$

где многочлены с рациональными коэффициентами $A_{0}(z), A_{1}(z)$ удовлетворяют условиям

$$
\operatorname{deg} A_{0}(z) \leqslant b_{1}-c_{1}-1, \quad \operatorname{deg} A_{1}(z) \leqslant b_{1}-a_{1}-1, \quad \operatorname{ord}_{z=1} A_{1}(z) \geqslant b_{1}-a_{1}-c_{1} .
$$

Отсюда следует (8) при $m=1$.

При $c_{1} \leqslant 0$ согласно предложению 1 имеем $A_{1}(z)=0$; в этом случае $d=0$, что согласуется с утверждением теоремы 2 . Итак, при $m=1$ утверждение теоремы выполняется.

Далее будем считать, что $m \geqslant 2$ и для интеграла $F_{m-1}(z)($ см. $(4))$ утверждение справедливо. В силу (4) имеет место соотношение

$$
F_{m}(z)=\frac{z^{b_{m}-b_{m-1}}}{\Gamma\left(b_{m}-a_{m}\right)} \int_{0}^{1} \frac{x^{a_{m}-b_{m-1}}(1-x)^{b_{m}-a_{m}-1}}{(1-z x)^{c_{m}}} F_{m-1}(z x) d x
$$

Согласно индуктивному предположению

$$
F_{m-1}(z)=\sum_{h=0}^{q} B_{h}(z) f_{h}(z)
$$

где $q$ есть количество положительных чисел среди $c_{1}, \ldots, c_{m-1}$, а многочлены с рациональными коэффициентами $B_{h}(z)$ удовлетворяют условиям

$$
\begin{gathered}
\operatorname{deg} B_{h}(z) \leqslant b_{m-1}-\min _{0 \leqslant k \leqslant m-1}\left(a_{k}+\sum_{i=k+1}^{m-1} c_{i}\right)-1 \\
\operatorname{ord}_{z=1} B_{h}(z) \geqslant \sum_{i=m-h}^{m-1}\left(b_{i}-a_{i}-c_{i}\right) .
\end{gathered}
$$


Другими словами, функция $F_{m-1}(z)$ представима как сумма слагаемых вида

$$
(1-z)^{k} f_{h}(z)
$$

где параметры $h, k$ удовлетворяют условиям

$$
0 \leqslant h \leqslant q, \quad \sum_{i=m-h}^{m-1}\left(b_{i}-a_{i}-c_{i}\right) \leqslant k \leqslant b_{m-1}-\min _{0 \leqslant k \leqslant m-1}\left(a_{k}+\sum_{i=k+1}^{m-1} c_{i}\right)-1 .
$$

Докажем, что при любых параметрах $h, k$, удовлетворяющих (22), функция

$$
H(z)=\frac{z^{b_{m}-b_{m-1}}}{\Gamma\left(b_{m}-a_{m}\right)} \int_{0}^{1} \frac{x^{a_{m}-b_{m-1}}(1-x)^{b_{m}-a_{m}-1}}{(1-z x)^{c_{m}-k}} f_{h}(z x) d x
$$

представима в виде

$$
H(z)=\sum_{j=0}^{h+1} C_{j}(z) f_{j}(z)
$$

где многочлены $C_{j}(z) \in \mathbb{Q}[z]$ удовлетворяют условиям (8). Применим предложение $1 \mathrm{k}$ интегралу (23), выбрав $a=a_{m}-b_{m-1}+1, b=b_{m}-b_{m-1}+1, c=c_{m}-k$. Согласно этому предложению имеет место тождество (24). Для многочлена $C_{h+1}(z)$ справедливо неравенство

$$
\operatorname{deg} C_{h+1}(z) \leqslant b-a-1=b_{m}-a_{m}-1 \leqslant b_{m}-\min _{0 \leqslant k \leqslant m}\left(a_{k}+\sum_{i=k+1}^{m} c_{i}\right)-1 .
$$

Если $0 \leqslant j \leqslant h$, то по предложению 1 и в соответствии с (22) имеем

$$
\begin{aligned}
\operatorname{deg} C_{j}(z) & \leqslant b-\min (a, c)-1=b_{m}-\min \left(a_{m}, c_{m}+b_{m-1}-k-1\right)-1 \\
& \leqslant b_{m}-\min \left(a_{m}, c_{m}+\min _{0 \leqslant k \leqslant m-1}\left(a_{k}+\sum_{i=k+1}^{m-1} c_{i}\right)\right)-1 \\
& =b_{m}-\min _{0 \leqslant k \leqslant m}\left(a_{k}+\sum_{i=k+1}^{m} c_{i}\right)-1 .
\end{aligned}
$$

Далее, при $1 \leqslant j \leqslant h+1$, пользуясь (22) и предложением 1 , находим

$\operatorname{ord}_{z=1} C_{j}(z) \geqslant b-a-c=b_{m}-a_{m}-c_{m}+k \geqslant \sum_{i=m-h}^{m}\left(b_{i}-a_{i}-c_{i}\right) \geqslant \sum_{i=m+1-j}^{m}\left(b_{i}-a_{i}-c_{i}\right)$.

Неравенства (25)-(27) означают справедливость (8) в тождестве из теоремы 2.

При $c_{m}>0$ неравенство $h \leqslant q$ означает, что $h+1$ непревосходит количества положительных среди чисел $c_{1}, \ldots, c_{m}$. Если $c_{m} \leqslant 0$, то при любом $k$ вьполняется $c_{m}-k \leqslant 0$, так что согласно предложению 1 в равенстве $(24)$ выполняется $C_{h+1}(z)=0$. Это значит, что параметр $d$ в теореме не превосходит $q$. Доказательство теоремы 2 этим завершается.

Настоящие результаты были получены во время моего пребывания в Университете г. Кёльна. Этому университету, а также профессору Петеру Бундшу, я хотел бы выразить мою глубокую благодарность за гостеприимство и творческую атмосферу. 


\section{СПИСОК ЦИТИРОВАННОЙ ЛИТЕРАТУРЫ}

[1] Зудилин В.В.Алгебраические соотношения для кратных дзета-значений // УМН. 2003. T. 58. № 1 . С. 3-32.

[2] Уланский Е. А. Тождества для обобщенных полилогарифмов // Матем. заметки. 2003. Т. 73. № 4. С. $613-624$.

[3] Сорокин В. Н. О теореме Апери // Вестн. МГУ. Сер. 1. Матем., мех. 1998. № 3. С. 48-53.

[4] Сорокин В.Н. О мере трансцендентности числа $\pi^{2}$ // Матем. сб. 1996. Т. 187. № 12. C. $87-120$.

[5] Нестеренко Ю. В. Интегральные тождества и конструкции совместных приближений к значениям дзета-функции Римана // Tруды IV Международной конференции "Современные проблемы теории чисел и ее приложения" (г. Тула, 2001 г.). М.: Изд. мех.-мат. фак-та МГУ, 2002. C. $115-132$.

[6] Rivoal T. La fonction zéta de Riemann prend une infinité de valeurs irrationelles aux entiers impairs // C. R. Acad. Sci. Paris Sér. 1 Math. 2000. V. 331. № 4. P. 267-270.

[7] Злобин С. А. Разложения кратных интегралов в линейные формы // Докл. РАН. 2004. Т. 398. № 5. C. $595-598$.

[8] Злобин С. А. Интегралы, представимые в виде линейных форм от обобщенных полилогарифмов // Матем. заметки. 2002. Т. 71. №5. С. 782-787.

[9] Mahler K. Ein Beweis des Thue-Siegelschen Satzes über die Approximation algebraischer Zahlen für binomische Gleichungen // Math. Ann. 1931. V. 105. P. 267-276.

[10] Baker A. Simultaneous rational approximations to certain algebraic numbers // Proc. Cambridge Philos. Soc. 1967. V. 63. P. 693-702.

[11] Mahler K. Zur Approximation der Exponentialfunction und des Logarithmus // J. Reine Angew. Math. 1932. V. 166. P. 118-150.

[12] Люк Ю. Специальные математические функции и их аппроксимации. М.: Мир, 1980.

[13] Jager H. A multidimensional generalization of the Pade table // Indag. Math. 1964. V. 26. P. 193-249. 